\title{
Harvesting Mandibular Ramus Bone Grafts Using Ultrasonic Surgical Device: Report of 20 Cases
}

\author{
Berkay Tolga SUER ${ }^{1 *}$ and Zekai YAMAN ${ }^{2}$ \\ ${ }^{1}$ Department of Oral and Maxillofacial Surgery, Haydarpasa Teaching Hospital, Istanbul, Turkey \\ ${ }^{2}$ Department of Oral Surgery, VKV American Hospital, Istanbul, Turkey
}

Received: January 23, 2014; Accepted: January 27, 2014; Published: January 28, 2014

"Corresponding author: Berkay Tolga SUER, Department of Oral and Maxillofacial Surgery, GATA Haydarpasa Teaching Hospital, Tibbiye Cad. Selimiye Mah. Uskudar, 34668, Istanbul, Turkey, Tel: +90 216 52422020-(4622); E-mail: berkaysuer@gmail.com

\begin{abstract}
Successful osseointegration of the dental implants requires sufficient bone volume surrounding implant site. The mandibular ramus is considered reliable donor site for reconstructing alveolar ridge defects prior to dental implant placement. The aim of this study was to present the clinical efficiency of using ultrasound surgery for harvesting mandibular ramus bone graft.

20 patients (10 male, 10 female; mean age $47.5 \pm 11.4$ years) with insufficient bone volume, who underwent autogenous bone grafting prior to implant placement, were presented. The grafts were harvested from the ascending ramus along with the external oblique ridge using an ultrasound surgical device (Piezosurgery, Mectron, Italy) from 20 donor sites and used to augment alveolar ridge atrophies. After waiting 4 to 5 months for healing grafted sites, 48 dental implants were inserted. Clinical parameters such as inferior alveolar nerve (IAN) damage, complications at donor and recipient site, the size of the graft and implant placement at the proper site were evaluated retrospectively. Mean graft length was $20.1 \mathrm{~mm}(\mathrm{SD}=2.73$ ), and mean graft width was $12 \mathrm{~mm}(\mathrm{SD}=2.47)$. Of 20 recipient sites, 20 healed uneventfully, and of 20 donor sites 18 healed uneventfully. Only one patient had transient altered sensitivity of the lower lip. Ultrasound surgery allowed precise and smooth bone cutting with better intraoperative visibility. Specially angulated tip of the devise made inferior horizontal cut easier and safer. The results of this study suggest that the use of ultrasound surgery is an effective method for harvesting bone grafts from mandibular ramus with avoidance of any major complication.
\end{abstract}

Keywords: Ultrasound surgery; Mandible; Osteotomy; Bone graft; Horizontal ramus

\section{Introduction}

Preprosthetic bone augmentation procedures such as implant site development using different kind of bone material are wellestablished and successful procedures. Placement of an implant in an optimal 3-dimensional position is a requisite for successful biological integration and prosthetic restorations. Restoration driven treatment protocols may dictates the placement of an implant in a certain localization that usually requires an augmentation procedure to increase the bone volume [1]. Although, different kinds of homogeneic, xenogenic or synthetic bone graft materials have been introduced, autogenous bone still considered as gold standard [2].

The choice of donor site is basically depend upon the quantity, quality and the form of the bone required. Typical intraoral donor sites for alveolar ridge augmentation are the ascending ramus, the mandibular body, symphysis and maxillary tuberosity. The advantages of harvesting intraoral autogenous bone for grafting include increased bone quality, easy access, close proximity of the donor and recipient sites, low morbidity, performed on outpatient basis and free from cutaneous scarring [3]. Mandibular ramus area is one of the most preferred donor sites with certain advantages among intraoral sites [4].

In general, the ramus area yields a rectangular piece of cortical bone approximately 2 to $4.5 \mathrm{~mm}$ thick, $1.5 \mathrm{~cm}$ in height and 3 $\mathrm{cm}$ in length [5]. This graft can provide adequate bone volume up to 3-tooth and even 4-tooth edentulous site for grafting [3]. According to study done by Gungormus M \& Yavuz MS [6], the average bone volume that could be harvested from anterior part of the ascending ramus was $2.36 \mathrm{~mL}$, and average surface area was $495.1 \mathrm{~mm}$. The mandibular ramus graft has been used for bone augmentation prior to implant placement [4,7-10], sinus grafting $[11,12]$, and immediate reconstruction following tumor surgery $[13,14]$.

Harvesting of the ramus block requires a rectangular shape bone cuts with motor-driven rotary or oscillating tools. Motordriven rotary or oscillating tools have potential to injure the soft tissues such as nerves and vessels. With this in mind, Khoury F et al. [2] have developed a special electric handpiece with a cutting disk for the harvesting of ramus bone grafts. Vercellotti et al. [15] have introduced a completely new bone cutting instruments called "Piezosurgery". Piezosurgery is the state of the art bone cutting instrument in oral surgery and its effectiveness in harvesting of the ramus bone graft was reported by Happe A [16].

Piezosurgery is an innovative technology that uses modulated ultrasonic frequency to cut mineralized tissue. Ultrasonic surgery device uses micro-oscillations of 60 to $200 \mu \mathrm{m} / \mathrm{sec}$ at $25-29 \mathrm{kHz}$. The micro-oscillations, which are created at this frequency, cut only mineralized hard tissue while adjacent soft tissue, nerves 
and vessels remain unharmed. Using ultrasonic surgery, it is possible to cut mineralized tissue with greater precision and selectivity. Cavitation effect that is created by the irrigation/ cooling solution and oscillating tip of the device, provides bloodfree surgical area, as a result greater visibility for the surgeon. With regard to bone formation and healing, it has been showed that ultrasound bone cutting is more favorable than it is with conventional bone cutting techniques such as carbide rotary instruments in experimental animal model [17].

The aim of present study was to clinical evaluation of the efficacy of ultrasound surgery for harvesting of bone grafts from mandibular ramus.

\section{Materials and Methods}

Twenty patients (10 females and 10 males) between ages of 23 and 62 years with inadequate bone volume for implant placement were included to this study. The mean age of the patients was $47.5 \pm 11.4$ years $(46.4 \pm 11.9$ years for the females, $48.6 \pm 11.5$ years for the males). All patients were in good health except for 4 patients who had controlled systemic diseases. Preoperative diagnoses were partial edentulouism with inadequate bone volume for implant placement. Therefore, bone augmentations of the implant site using mandibular ramus bone grafts were planned. Patients were informed about surgery, postoperative recovery and possible complications and they signed an informed consent form. Before surgeries panoramic radiographs and cone beam computerized tomographic scans (in five patients) were obtained to evaluate donor and recipient sites. All of the patients underwent the procedure unilaterally and harvested ramus grafts used either the maxilla or mandible. As a result, 20 sites were grafted in 20 patients and 48 dental implants were inserted.

\section{Surgical technique}

All procedures performed under local anesthesia. Surgical approach to the ramus area was performed as described by Misch CM [4] and according to the recipient site defects suitable amount of bone graft was harvested. All bone-related surgeries were performed using Piezosurgery device (Mectron, Italy) with saw shape inserts (Figure 1). For ramus bone harvesting, four osteotomies were made: one superior and 2 vertical as well as one osteotomy was made at the inferior border (Figure 2). The superior horizontal cut was made 4 to $5 \mathrm{~mm}$ medial to the external oblique ridge with an OT7 insert (principal micro-saw) that has a measure to control the depth of the cut (Figure 3).

With OT7 insert, cut was made from the first or second molar region and continues posteriorly along the external oblique ridge to ascending ramus depend upon the required size. The two vertical osteotomies, anterior and posterior, were also made with the OT7 insert extending 10 to $12 \mathrm{~mm}$ in length in the superoinferior direction depending on the needed size (Figure 4). Then, a cut connecting the inferior aspect of each vertical osteotomy was made with the angulated bone saw inserts OT8R and OT8L insert depending on the side that graft was taken. This special insert was exclusively produced for this

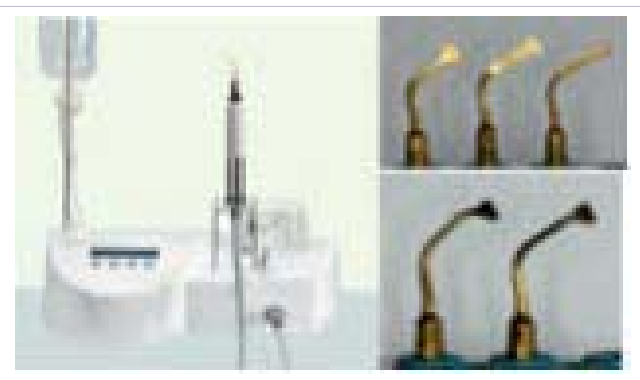

Figure 1: Piezosurgery (Mectron 3, Genoa, Italy) and inserts were used in this study. Piezoelectric device uses modulated micro-vibrations of $60-200 \mu \mathrm{m} / \mathrm{s}$ at $24-29 \mathrm{kHz}$. Different piezoelectric inserts were used for bone osteotomies (OT2, OT7, OT8L).

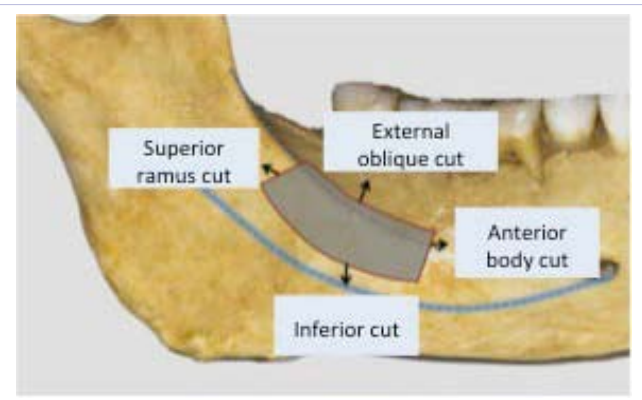

Figure 2: The area marked in gray represent the ideal site for harvesting ramus graft. Black arrows showing two vertical and two horizontal osteotomy lines for harvesting ramus graft (Misch 2008).
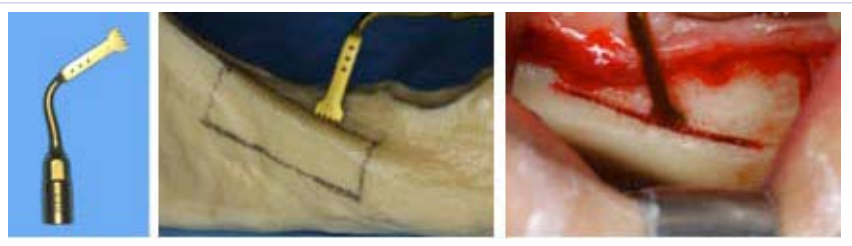

Figure 3: OT7 insert with scale for depth control is used for different bone cuts in ramus bone grafting such as external oblique, superior ramus and anterior body cuts. Schematic and clinical picture shows using OT7 insert for superior horizontal cut.
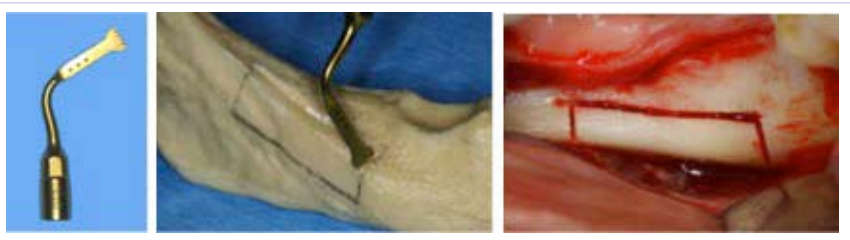

Figure 4: OT7 insert is used for anterior body and superior bone cuts. Surgical cuts made with a piezoelectric surgery prior to pry out the ramus block graft.

inferior horizontal cut (Figure 5). After completed all the outline cuts of the graft, the harvest was usually pried out by gentle manipulation with a small flat chisel and mostly without using hammer. However in this presented study $5(75 \%)$ of the cases needed to use hammer for mobilizing buccal plate (Figure 6). To prevent complications such as post-operative bleeding and hematoma caused by excessive bleeding of the donor site and 
surrounding soft tissues, the surgical site was inspected properly and hemostasis was achieved with cauterization before primary suturing. The donor site was primarily sutured back with 3-0 Vicry ${ }^{\circledR}$ suture.

All patients received a single preoperative dose of oral antibiotics amoxicillin/clavulanate sodium (Augmentin ${ }^{\circledR}$ BID, 1 gram, Glaxo-Smith-Kline, Turkey) or clindamycin (Klindan 150 mg; BilimIlac, Turkey) that same antibiotic regimen continued for 5 days postoperatively. Additionally, nonsteroidal antiinflammatory agent naproxen sodium (Apranax Forte ${ }^{\circledR} 500 \mathrm{mg}$,) for pain and swelling, were prescribed as needed. Patients were also advised to rinse three times per day with $0.2 \%$ concentration chlorhexidine (Klorhex, Drogsan, Turkey) mouthwash for 7 days post-operatively. Extraoral application of a cold pack was recommended for 12 hours after the surgery.

The harvested monocortical bone block were used for usual bone grafting procedures such as lateral or onlay grafting, and vertical bone grafting for further dental implant placement (Figure 1-6). Block grafts size was measured upon harvested from donor site with the surgical caliper. All postoperative complications either at parent or recipient site were recorded. Hematoma or wound dehiscence without infection considered minor complications, while bleeding, changing in sensitivity and infection were considered as major complications.

After waiting 4-5 monthsfor bony healing, mucoperiosteal flaps were raised for placing dental implants. All grafts were evaluated clinically regarding incorporation to the parent bone. All subjects received dental implant according to preoperative prosthetic driven ideal sites. Graft procedure was considered successful if the ideal dental implant insertion was achieved.

\section{Results}

All patients recovered well after the operations. All of the surgeries were performed as planned and there was no intraoperative surgical complication.
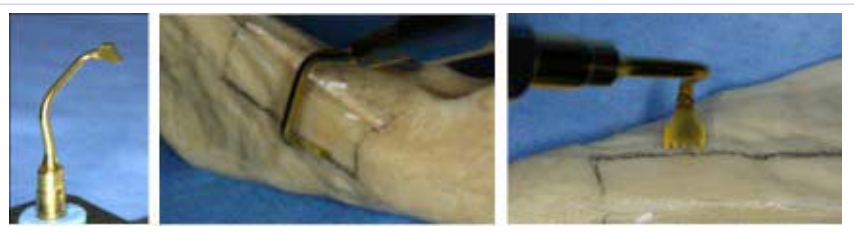

Figure 5: OT8 R insert is used for horizontal bone (inferior cut) cuts. Mandibular model showing orienting the OT8 piezoelectric insert for inferior horizontal cut.
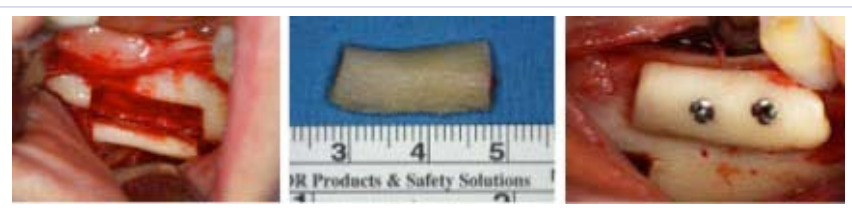

Figure 6: The ramus block graft was out fractured with chisels via gentle tapping from mandible. The trimmed block graft was fixed in recipient site with miniscrews.
Twenty donor sites were treated with autogenous ramus bone graft in 20 patients ( 10 male, 10 female). Of 20 patients six were current smoker ( $<10$ cigarettes a day) that $30 \%$ of patients. A total of 20 sites were grafted using different techniques such as horizontal veneer grafting $(n=8,90 \%)$, and vertical grafting $(n=2,10 \%)$. Guided bone regeneration (GBR) membranes used for all cases. Of these 2 grafted sites, $25 \%(n=5)$ was in maxilla and $75 \%(n=15)$ was in mandible. A total of 48 dental implants were inserted in grafted sites; 25 were placed in women (52\% of implants) and 23 were inserted in men (48\% of implants).

The mean length of the obtained graft was $20.1 \mathrm{~mm}$ $(\mathrm{SD}=2.73 \mathrm{~mm})$, and the mean width of the obtained graft was $12 \mathrm{~mm}(\mathrm{SD}=2.47 \mathrm{~mm})$. Seventeen of the 20 donor sites healed uneventfully (90\%), whereas 2 of these healed with complications (15\%). Of these 2 of the complications, one was wound dehiscence without any infection (5\%), and one was altered sensitivity of the right lower lip (5\%) that resolved after 2 months. As a result, all complications were healed without causing any further impairment for the patients.

\section{Discussion}

Ultrasound surgery has certain advantages over traditional manual or high-speed motorized instruments in oral and maxillofacial surgery. Micro-oscillations of the tip of the devise, operates in low frequency range between $24-29 \mathrm{kHz}$, allows for precise cutting and yields minimal wastage of bone [14, 18]. Frequencies lower than $50 \mathrm{kHz}$ causes minimal damage to soft tissues (nerves, vessels, mucosa) [19]. The unique phenomenon of cavitation effect gives operator a better visibility than using any conventional manual or rotary instrument. This air cavitation also reduces the hemorrhage at surgical site. Due to positive features mentioned above, ultrasound surgery devices have gained widespread acceptance in dentistry [20,21]. These findings are confirmed in this presented study by the author's experiences.

One of the important difficulties harvesting ramus block graft mentioned in literature is that managing the caudal horizontal cut due to close proximity of the IAN. In addition, due to tight attachments of the masseter and buccal muscle and close proximity of the facial artery at this level make this caudal horizontal cut difficult to see and manage with the conventional instrumentation. Before ultrasonic surgery was introduced, in conventional technique, authors recommended making grooves at inferior osteotomy line instead of full cortical cut to avoid damaging IAN [3]. IAN complications of harvesting bone from ramus and symphysis using conventional instrumentation are variable in the literature. In one clinical study, authors reported that, even after 18 -month-healing period, $52 \%$ of the symphysis group had decreased sensitivity, whereas the ramus group had only $3 \%$ decreased sensitivity with the permanent altered sensitivity in the posterior vestibular area [22]. With the specially angulated OT8R or OT8L insert of the device, ultrasound surgery has distinct advantage over conventional technique. Using this special tip for horizontal cut, surgeon does not need to reflect the flap extensively and making complete caudal horizontal 
cut is possible without damaging IAN. Having made complete caudal horizontal cut, it is easy to pry out ramus graft with gentle manipulation with the chisel. In that way the unwanted side effect, paresthesia of the IAN, seems to be limited and temporary. Complications of IAN with ultrasonic surgery seems less compared to conventional techniques in the literature. According to clinical study done by Happe A [16] one patient reported altered IAN sensitivity out of 45 ramus graft harvest, which had lasted 6 months. In this presented study, of 20 ramus bone graft harvest, only one patient had IAN disturbance lasted 2 months. Our IAN complication rate seems to be relatively low and comparable to the results of studies concerning intraoral harvesting in the literature $[2,8,16,22,23]$. With regard to IAN visibility during harvesting procedure, Alfaro FH [23] reported that the IAN was visible about $10 \%-12 \%$ of the cases. In this study, IAN was visible three out of 20 cases, which is also consistent with the previous reports. According to author's clinical experience, being able to prepare complete cut at the inferior level with the specially designed OT8 insert allow clinician to avoid excessive usage of chisel and hammering, which then results less complications.

As with the regenerative surgeries such as bone augmentation, the effect of instruments that used for harvesting bone on structure of bone and viability of cells are of great importance. In an animal study, researchers suggested that piezoelectric surgery yielded faster bone healing and remodeling [24]. In a clinical study, researchers used histomorphometric evaluation to compare efficiency of different methods to harvest bone grafts. Their results showed that piezoelectric surgery was one of the best methods of harvesting bone graft along with gouge-shaped bone chisel, back-action, en block harvesting, and rongeur pliers. They also noted that the bone harvested with round bur on lowspeed hand-piece, bur on high-speed hand-piece, spiral implant bur, or safe scraper are not suitable for grafting as indicated by the absence of osteocytes and the predominance of non-vital bone [25].

With regard to postoperative trismus after ramus bone harvesting, using conventional techniques, Sittitavornwong S \& Gutta R [3] reported that the rate seems to be high (60\%) but transient and can be lasted 3-4 weeks to resolve. In this study only two patients had trismus after surgery $(10 \%)$ that lasted 2 weeks to resolve. Our result is in contrast to this study. However, Happe A [16] reported none out of 45 harvest using piezosurgery. When compared to this study, our results shoved comparable results.

Happe A [16] reported that infection rates after harvesting bone from ramus is $2.22 \%$. In this study infection was not seen in any patient in any site (donor or recipient). Previous study reported that out of 45 ramus bone harvest with ultrasonic surgery only one dehiscence without abscess (2.22\%) occurred. In this study, similarly, only one patient had wound healing (5\%) Overall, Happe A [16] stated that $93.3 \%$ of the cases healed without complications. In this study our results shoved $90 \%$ of the cases healed without complication which similar to his report.

In this study recipient sites showed good performance. All pre-planned implants were inserted and osseointegrated well.
In conclusion, with similar to previous reports ultrasonic surgery yields to precise and clean harvest graft from ramus without harming IAN. Therefore authors of this study strongly suggest using ultrasonic surgery for this purpose.

\section{References}

1. Garber DA, Belser UC (1995) Restoration-driven implant placement with restoration-generated site development. Compend Contin Educ Dent 16(8): 796, 798-802, 804

2. Khoury F, Khoury C (2007) Mandibular bone block grafts: Diagnosis, instrumentation, harvesting techniques and surgical procedures, in Bone Augmentation in Implant Dentistry. In F Khoury, H Antoun \& P Missika (eds.), Quintessence Publishing, USA, pp. 115-212.

3. Sittitavornwong S, Gutta R (2010) Bone graft harvesting from regional sites. Oral Maxillofac Surg Clin North Am 22(3): 317-330.

4. Misch CM (1996) Ridge augmentation using mandibular ramus bone grafts for the placement of dental implants: presentation of a technique. Pract Periodontics Aesthet Dent 8(2): 127-135.

5. Li KK, Schwartz HC (1996) Mandibular body bone in facial plastic and reconstructive surgery. Laryngoscope 106(4): 504-506.

6. Gungormus M, Yavuz MS (2002) The ascending ramus of the mandible as a donor site in maxillofacial bone grafting. J Oral Maxillofac Surg 60(11): 1316-1318.

7. Nowzari H, Aalam AA (2007) Mandibular cortical bone graft part 2: surgical technique, applications, and morbidity. Compend Contin Educ Dent 28(5): 274-280.

8. Misch CM (1997) Comparison of intraoral donor sites for on lay grafting prior to implant placement. Int J Oral Maxillofac Implants 12(6): 767-776.

9. Jensen J, Sindet-Pedersen S, Oliver AJ (1994) Varying treatment strategies for reconstruction of maxillary atrophy with implants: results in 98 patients. J Oral Maxillofac Surg 52(3): 210-216.

10. Aalam AA, Nowzari H (2007) Mandibular cortical bone grafts part 1: anatomy, healing process, and influencing factors. Compend Contin Educ Dent 28(4): 206-212.

11. Wheeler SL, Holmes RE, Calhoun CJ (1996) Six-year clinical and histologic study of sinus-lift grafts. Int J Oral Maxillofac Implants 11(1): 26-34.

12. Lundgren S, Moy P, Johansson C, Nilsson H (1996) Augmentation of the maxillary sinus floor with particulated mandible: a histologic and histomorphometric study. Int J Oral Maxillofac Implants 11(6): 760766.

13. Muto T, Kanazawa M (1997) Mandibular reconstruction using the anterior part of ascending ramus: report of two cases. J Oral Maxillofac Surg 55(10): 1152-1156.

14. Yaman Z, Suer BT, Cebe P, Keles M (2011) Piezosurgical excision of a large maxillary odontoma, in XVII Congresso Nazionale della Societa Italiana Chirurgica Maxillo-Facciale (SICMF). In P Ronchi (ed.) Medimond International Proceedings: Como, Italy, pp. 417-421.

15. Vercellotti T, Nevins ML, Kim DM, Nevins M, Wada K, et al. (2005) Osseous response following resective therapy with piezosurgery. Int J Periodontics Restorative Dent 25(6): 543-549.

16. Happe A (2007) Use of a piezoelectric surgical device to harvest bone grafts from the mandibular ramus: report of 40 cases. Int J Periodontics Restorative Dent 27(3): 241-249. 
17. Vercelotti T (2009) Essentials in Piezosurgery: Clinical advantages in dentistry. Quintessenza Edizioni, Milano, Italy, p. 22.

18. Yaman Z, Suer BT (2013) Piezoelectric surgery in oral and maxillofacial surgery. Annals of Oral and Maxillofacial Surgery 1(1): 5.

19. Nusrath MA, Postlethwaite KR (2011) Use of piezosurgery in calvarial bone grafts and for release of the inferior alveolar nerve in sagittal split osteotomy: technical note. Br J Oral Maxillofac Surg 49(8): 668669.

20. Pavlikova G, Foltan R, Horka M, Hanzelka T, Borunska H et al. (2011) Piezosurgery in oral and maxillofacial surgery. Int J Oral Maxillofac Surg 40(5): 451-457.

21. Yaman Z, Suer BT (2013) Clinical comparison of ultrasonic surgery and conventional surgical techniques for enucleating jaw cysts. Int J Oral Maxillofac Surg 42(11): 1462-1468.
22. Clavero J, Lundgren S (2003) Ramus or chin grafts for maxillary sinus inlay and local onlay augmentation: comparison of donor site morbidity and complications. Clin Implant Dent Relat Res 5(3): 154160.

23. Alfaro FH (2006) Bone grafting in oral implantology: techniques and clinical applications. (1stedn), Quintessence Publishing, Barcelona, Spain.

24. Ma L, Stubinger S, Liu XL, Schneider UA, Lang NP (2013) Healing of osteotomy sites applying either piezosurgery or two conventional saw blades: a pilot study in rabbits. Int Orthop 37(8): 1597-1603.

25. Berengo M, Bacci C, Sartori M, Perini A, Della Barbera M, et al. (2006) Histomorphometric evaluation of bone grafts harvested by different methods. Minerva Stomatol 55(4): 189-198. 